\title{
Adrenal Insufficiency
}

\author{
ASHRAFUZZAMAN SM ${ }^{\mathrm{a}}$, RAHIM MA ${ }^{\mathrm{b}}$
}

\begin{abstract}
Adrenal insufficiency means hypo-function of the adrenal cortex, usually glucocorticoid, may be associated with mineralocorticoid and rarely adrenal androgen. It can be categorized into two types: Primary adrenal insufficiency, or Addison's disease (cortisol low and ACTH high) or secondary/ tertiary adrenal insufficiency due to pituitary or hypothalamic disorders (both cortisol and ACTH are low). Identification of etiology is very important for plan of treatment. The reported incidence of adrenal insufficiency varies greatly depending on the population studied, the test and cut off levels used, and the cause. Many patients may present with adrenal crisis, some of them have subtle symptoms, hyperpigmentation, hypotension, electrolyte imbalance, shock etc. Many of them have iatrogenic adrenal insufficiency with
\end{abstract}

\section{Introduction}

Adrenal insufficiency means hypo-function of the adrenal cortex usually glucocorticoid (cortisol) insufficiency. This may be associated with mineralocorticoid (aldosterone) and androgen (DHEAS) insufficiency. The clinical features are usually variable depending on whether the onset is acute, chronic or with adrenal crisis. ${ }^{1}$ Adrenal crisis should be considered when patients present with vascular collapse (shock) whether or not known to have adrenal insufficiency. Sometimes these patients are having features of Cushing's syndrome due to chronic steroid use. Some of the patients may present with non-specific symptoms (like fatigue, weakness, anorexia, weakness) etc. Unexplained hyponatremia, hypoglycemia, weight loss should be suspected for chronic adrenal insufficiency.

a Dr. S.M. Ashrafuzzaman, MD (Endocrinology), FACE (USA), Associate Professor, Department of Endocrinology, BIRDEM General Hospital, Dhaka, Bangladesh.

b Dr. Muhammad Abdur Rahim, FCPS (Medicine), Assistant Professor, Department of Nephrology, BIRDEM General Hospital, Dhaka, Bangladesh.

Address of Correspondence: Dr. S.M. Ashrafuzzaman, MD (Endocrinology), FACE (USA), Associate Professor, Department of Endocrinology, BIRDEM General Hospital, Dhaka, Bangladesh. Email: ashraf_zaman1961@yahoo.com.

Received: Nov 11, 2014

Accepted: 20 April, 2015 features of Cushing's syndrome. So, the manifestations of adrenal insufficiency are numerous and nonspecific, and clinicians should have a high index of suspicion and be alert to see important diagnostic clues, such as hyponatremia, hyperkalemia, and hypotension (that are refractory to fluids and vasopressors), diarrhea, weight loss, vomiting, fever etc. without any clear causation. Treatment of adrenal insufficiency is usually life-long, of course based on etiology. Short acting glucocorticoid is better, like hydrocortisone. Patients may be treated with prednisolone. Patients should be provided a "steroid card". Sometimes it is essential to take patients under "withdrawal protocol".

Key words: Addison's disease, adrenal gland, adrenal insufficiency.

(Birdem Med J 2015; 5(Supplement 1): 43-48)

Primary adrenal insufficiency refers to disease of the adrenal gland itself. Secondary adrenal insufficiency is due to deficiency of ACTH of anterior pituitary rarely may be due to $\mathrm{CRH}$ deficiency due to hypothalamic disease. Minerelocorticoid deficiency invariably accompanies primary hypoadrenalism but does not accompany secondary adrenal insufficiency. In case of secondary adrenal insufficiency, renin-angiotensinaldosterone axis remains intact. Another important cause of both glucocorticoid and minerelocorticoid deficiency is found in congenital adrenal hyperplasia $(\mathrm{CAH}){ }^{2}$

\section{Primary adrenal insufficiency}

Thomas Addison described this condition in his classical monograph published in $1855 .^{3}$ The condition occurs in 4-11 cases per 100,000. In our experience it is not very rare, though we do not know the exact prevalence rate in Bangladesh. The disease is associated with significant morbidity and mortality but if diagnosed can easily be treated. ${ }^{1,4}$

The common causes of primary adrenal insufficiency Infections (Tubercular, Fungal, HIV, CMV etc).

Others are autoimmune (may be sporadic or may be with polyglandular syndrome Type I: Addison's disease, mucocutaneous candidacies, hypoparathyroidism, dental problem, primary gonadal failure, alopecia etc). 
Autoimmune Polyglandular Syndrome (APS) Type II: (Schmidt's Syndrome): (Addison's disease, primary hypothyroidism, primary hypogonadism, Type 1 Diabetes mellitus, vitiligo, Pernicious anemia, alopecia, ITP, RA etc).

Other rare causes are: Metastatic (from lung, breast), Infiltrative (hemachromatosis, amyloid, lymphoma), $\mathrm{CAH}$, adrenal surgery (bilateral adrenalectomy for Cushing's), adrenal hemorrhage, drugs (metyrapone, ketoconazole etc).

The common causes of secondary adrenal insufficiency

These are glucocorticoid withdrawal, pituitary or hypothalamic disorders (hypopituitarism, apoplexy, Sheehan's syndrome, surgery, radiation) etc.

\section{Presentation}

Clinical features of glucocorticoid deficiency include weight loss, anorexia, weakness, lathery, nausea, vomiting, diarrhea, hypotension, dehydration, shock, hypoglycemia, hyponatremia, hyperkalemia, rarely hypercalcemia. Occasionally patients may present with pigmentation of sun exposed areas, mucous membrane, new scar etc due to high ACTH of primary adrenal disease. Rarely patient complaints of decreased body hair, loss of libido (female) due to adrenal androgen deficiency.

Adrenal insufficiency should be suspected in cases where there is history of prolonged use of glucocorticoid (more than 3 weeks). This usually happens with supraphysiological doses but may happen in patients with pharmacological dose and sudden withdrawal. Pigmentation is not always present. ${ }^{5}$ Hyperpigmentation is absent in some patients with primary adrenal insufficiency and in all patients with secondary (pituitary) and tertiary (hypothalamic) adrenal insufficiency. Sometimes other possibilities are perused when patient presents with gastrointestinal symptoms like diarrhea, vomiting, weight loss, anorexia etc. Even when the diagnosis appears obvious, endocrine evaluation is indicated to confirm the diagnosis and to determine the type of adrenal insufficiency and it's cause. However, therapy should be started before the diagnosis is established in an acutely ill patient with possible adrenal crisis. ${ }^{3}$

\section{Laboratory Diagnosis}

The diagnosis usually is done in 3 steps. $^{3}$

1. Demonstrating inappropriately low cortisol secretion (preferably by stimulation test)
2. Determining whether the cortisol deficiency is dependent on or independent of ACTH deficiency (primary or secondary) and evaluating minerelocorticoid secretion status in patients without ACTH deficiency (only in primary).

3. Seeking a treatable cause of the primary disorder e.g, Tuberculosis (TB), autoimmunity or fungal infection e.g. histoplasmosis involving the adrenal glands or a secondary disease e.g. pituitary adenoma/ apoplexy / post surgery, post infective etc compromising normal pituitary function). ${ }^{6}$

After establishing cortisol deficiency, lateralization is essential that whether it is due to primary of secondary. If ACTH is high it is primary or if ACTH is low along with low cortisol it is secondary adrenal insufficiency. Then comes the question of etiology of primary or secondary. Other tests to establish etiology include imaging of adrenal gland to see calcification, establishing infective cause like test for TB, fungus, HIV and rarely FNAC to see secondary. Tests for autoimmunity of other associated glands are essential if autoimmune Addison's disease is suspected.

Among the primary disorders most common is autoimmune, adrenal auto-antibody can be detected in $60-70 \%$ cases. Among the secondary causes most common is glucocorticoid withdrawal, drug history and sometimes clinical features of glucocorticoid excess is diagnostic (phenotypically Cushing, biochemically hypoadrenalism).

The diagnosis of adrenal insufficiency of any cause depends entirely upon the demonstration of inappropriately low cortisol production. Caution should be taken in patients with cirrhosis, nephrotic syndrome, or those taking oral estrogens. ${ }^{7,8}$ In these settings, decreased or increased levels may lead to an incorrect diagnosis. Salivary cortisol or free serum cortisol may be the alternative tests.

Controversies of S. cortisol (basal/random): Usually S. cortisol has it's peak and trough level due to stimulus of highly pulsatile ACTH/CRH. So, basal or random S. cortisol does not truly reflect the adrenal reserve and may give false positive result. So, it is always correlated with clinical feature and adrenal insufficiency is highly suspected if S. cortisol $<140 \mathrm{nmol} / \mathrm{L}(5 \mathrm{mcg} / \mathrm{dl}) .{ }^{9}$ 


\section{Procedure}

Synacthen test/ACTH stimulation test: Test can be done any time of the day, irrespective of last meal. Baseline (0 minute) $\mathrm{S}$. cortisol and ACTH blood sample is drawn. The $250 \mathrm{mcg}$ in $1 \mathrm{ml}$ Synathen injection is given IV or IM. The plasma cortisol sample at 30 minutes and $60 \mathrm{~min}$ are drawn for test. S. cortisol (total) is readily available and standardized in many laboratories but reliable ACTH test may not readily available.

\section{Standard dose test $(250 \mathrm{mcg})^{10}$}

A normal response to the high-dose (250 mcg as an IV bolus) ACTH stimulation test is a rise in serum cortisol concentration after either 30 or 60 minutes to a peak of $>18$ to $20 \mathrm{mcg} / \mathrm{dL}$ ( 500 to $550 \mathrm{nmol} / \mathrm{L}$ ). Conversion factor: microgram $/ \mathrm{dl} \times 28=\mathrm{nmol} / \mathrm{L}$.

A normal response to the high-dose (250 mcg) ACTH stimulation test excludes primary adrenal insufficiency and most patients with secondary adrenal insufficiency.

\section{Low dose test $(1 \mathrm{mcg})^{11,12}$}

The low-dose (1 mcg as an IV bolus) ACTH stimulation test criteria for a normal cortisol response after 20 or 30 minutes are more variable: 17 to $22.5 \mathrm{mcg} / \mathrm{dL}$ (400 to 620 $\mathrm{nmol} / \mathrm{L})$.

As $1 \mathrm{mcg}$ preparation is not readily available we have to dilute $250 \mathrm{mcg}$ in the following way:

At first $250 \mathrm{mcg}$ of $1 \mathrm{ml}$ injection is mixed with $24 \mathrm{ml}$ distilled water (DW). From this $25 \mathrm{ml}$ solution $1 \mathrm{ml}$ is taken in a syringe which contains $10 \mathrm{mcg}$ ACTH. Now this $1 \mathrm{ml}$ is dissolved with new $9 \mathrm{ml} \mathrm{DW}$. From this new $10 \mathrm{ml}$ each $1 \mathrm{ml}$ contains $1 \mathrm{mcg}$ Synacthen (ACTH, Cosyntropin).

The low dose test may have advantages over the standard high dose test in some clinical settings, in particular, for the diagnosis of recent onset $\mathrm{ACTH}$ deficiency (secondary adrenal insufficiency) and chronic partial pituitary ACTH deficiency 13 to avoid overstimulation with high dose $(250 \mathrm{mcg})$. But disadvantages are peak may be earlier at 15-20 minutes, and patients whose surface area varied significantly from $1.73 \mathrm{~m}^{2}$, it should be used with caution.

\section{Prolonged high-dose ACTH stimulation test (1 mg)}

Usually not done now-a-days. It helps in differentiating primary (adrenal) from secondary (pituitary) or tertiary (hypothalamic) disease. One $\mathrm{mg}$ (not the $250 \mathrm{mcg}$ ) Synacthen 6 hourly for 48 hours is given and $\mathrm{S}$. cortisol is measured daily to see increment. In secondary and tertiary adrenal insufficiency there is progressive increase but not in primary disease. To differentiate secondary from tertiary disease, $\mathrm{CRH}$ test can be done but rarely in clinical practice.

Another method of high dose test is $1 \mathrm{mg}$ Depot Injection Synacthen is given IM at 9 AM. S. cortisol samples are collected before and at 1, 2, 4, 6, 8 and 24 hours after injection.

It is very important that patients those are having steroid it is better to bring down at physiological dose, wait for few weeks or months according to dose and duration of use and then advice for Synacthen test. When glucocorticoid can not be stopped (patient on physiological dose of steroid), equivalent dose of dexamethason $(0.5-1 \mathrm{mg})$ should be given for at least one day prior to the test.

Table I

Comparison of commonly used glucocorticoid:

Equivalent Anti Infl- Mineralo Duration Dose mg ammatory corticoid of Action

\begin{tabular}{lcccc} 
& & Activity & Activity & (Hours) \\
\hline Hydrocortisone & 20 & 1 & 1 & $8-12$ \\
Prednisolone & 05 & 4 & 0.8 & $12-36$ \\
Dexamethason & 0.75 & 30 & 0 & $36-72$ \\
Triamcinolone & 4 & 5 & 0 & $12-36$ \\
Fludrocortisones & $\mathrm{X}$ & 10 & 125 & $12-36$ \\
\hline
\end{tabular}

(Courtesy: Up To Date 14, Graphic 64138, 2014).

\section{Adrenal insufficiency among critically ill patients}

Endocrinologists are frequently asked to evaluate adrenal function in intensive care unit (ICU) patients or role of steroid in ICU patient. Assessment of adrenal function at this situation is extremely difficult due to profound and variable changes in HypothalamoPituitary-Adrenal (HPA) axis. ${ }^{11}$ The critical care community is also interested to know about acquired adrenal insufficiency during critical illness. There are still limited evidence and also controversial, to identify and to manage corticosteroid insufficiency in this setting. Confirm diagnosis is also difficult by single test. ${ }^{14}$ But it is known that both high and low cortisol is associated with poor prognosis. ${ }^{9}$ Considering all caveats, assessment of adrenal insufficiency in critically ill patients are made on the basis of random cortisol or 
short ACTH stimulation test. ${ }^{15,16}$ Sometimes the term is used as relative adrenal insufficiency or critical illness related cortisol deficiency. Cortisol assay (Basal/ Random/ACTH stimulated) should be done only in highly suspected cases even with septic shock or ARDS. Clinicians should have a high index of suspicion for its occurrence in critically ill patients with persistent hypotension despite adequate fluid resuscitation and/ or poor hemodynamic response to vasopressor. ${ }^{18}$ Careful history taking and physical examination, complemented by other signs such as hyponatremia and hyperkalemia, should help identify most cases. ${ }^{16}$ Treatment should be given with hydrocortisone 100$200 \mathrm{mg}$ per day IV in divided doses for 5-7 days only.

\section{Adrenal insufficiency among children}

Adrenal insufficiency is rare in children. Patients usually present with GI symptoms, pigmentation, hypotension, hyperkalemia, hyponatremia etc. Adrenal crisis or acute adrenal insufficiency can be observed as the initial presentation of adrenal insufficiency or as the result of inadequate replacement therapy in pediatric patients with known adrenal insufficiency. ${ }^{3}$ To minimize morbidity and mortality, prompt recognition and treatment of adrenal crisis is critical. Diagnosis can be made by same procedure as adults. In the case of primary adrenal insufficiency of children, adrenal androgens (17-hydroxy progesterone) should be measured to evaluate for congenital adrenal hyperplasia. This is the most common cause of primary adrenal insufficiency but usually presents during infancy. Regarding autoimmune polyglandular syndrome (APS), antibodies to the adrenals and other endocrine glands may establish an autoimmune mechanism as adults. Children require hydrocortisone replacement, approximately 10 to $24 \mathrm{mg} /$ $\mathrm{m}^{2}$ per 24 hours when given orally. Best glucocorticoid is hydrocortisone in children. Patients with primary adrenal insufficiency also require minerelocorticoid replacement usually at higher dose than adults. Dose should be titrated according to blood pressure, electrolytes etc.

\section{Adrenal insufficiency and pregnancy}

Rarely pregnancy is complicated by adrenal insufficiency. Usually known adrenal insufficient patient was discouraged to be pregnant before, and before the advent of glucocorticoid, there was increased mortality of both mother and fetus. At present, most women adequately treated for adrenal insufficiency go through pregnancy, labor, and delivery without difficulty, and babies achieve a normal birth weight. The usual glucocorticoid and minerelocorticoid replacement doses are continued; an occasional woman requires slightly more glucocorticoid in the third trimester. ${ }^{3}$ Adrenal crisis is very rare except in first trimester. Hydrocortisone is the preferred agent, but usually given 2-3 times in divided doses at $50 \%$ at morning, $25 \%$ at afternoon and $25 \%$ at evening of daily total dose. During labor/delivery (NVD or CS) injectable hydrocortisone should be used. After delivery dose should be tapered to maintenance dose in next 2-3 days.

\section{Association of other endocrine autoimmune disease with autoimmune adrenalitis: APS I and APS II (Adrenal Insufficiency)}

Interestingly patients with APS are usually female (70\%), conversely isolated autoimmune adrenal insufficiency more common in males. In one series it was found that ${ }^{2,19}$, $53 \%$ of autoimmune adrenal insufficiency patients do not have other autoimmune disorders. Others may have ovarian (20\%), testicular (2\%), parathyroid (10\%), thyroid (Hypo-, Euthyroid and Hyperthyroid) (22\%), pernicious anemia (5\%), type 1 diabetes (11\%). Along with autoimmune adrenal insufficiency APS type I or APS type II are not uncommon. APS I includes hypothyroid, hypoparathyroid, mucocutaneous candidacies, type 1 diabetes, pernicious anemia, hypopituitarism, primary hypogonadism, autoimmune hepatitis, vitiligo etc. APS type II includes hypothyroid, type 1 diabetes, hypogonadism, diabetes insipidus, vitiligo, alopecia, pernicious anemia, myasthenia gravis, immune thrombocytopenia purpura, Sjogren's syndrome, rheumatoid arthritis etc. Autoimmune marker(s) should be tested among high indexed cases.

\section{Management of adrenal crisis}

When acute adrenal insufficiency (Addisonian Crisis) is suspected (circulatory collapse, hypotension, dehydration, nausea, vomiting, hypoglycemia) a blood sample is drawn immediately for random plasma cortisol and ACTH (if not diagnosed previously) establish IV line and start normal saline. Without waiting for laboratory results start hydrocortisone IV $100 \mathrm{mg} 6$ hourly. ${ }^{2}$ Add others supportive treatment. Depending on clinical response gradually tapper the dose, switch orally and gradually bring down to physiological dose. Justify minerelocorticoid use after reduction of IV dose 
(start if hypotension, hyponatremia, hyperkalemia, low plasma renin activity) only for primary adrenal insufficiency. Identify precipitating cause and treat accordingly. Take necessary steps to prevent recurrence.

\section{Management of chronic adrenal insufficiency/chronic} replacement therapy

Most common maintenance therapy is given by either hydrocortisone or by prednisone. Oral hydrocortisone is usually given 2-3 times, two-third of dose at early morning to mimic normal physiological glucocorticoid secretion and prevention of iatrogenic Cushingoid features as well as other metabolic complications like diabetes, dyslipidemia, hypertension, hypokalemia, weight gain, osteoporosis etc. Usually hydrocortisone is maintained $20 \mathrm{mg}$ at morning and $10 \mathrm{mg}$ at evening. Slight higher or better lower dose can be required in few patients. For prednisolone, as treatment of chronic adrenal insufficiency, usually given once daily, though previously practiced twice daily. Usual dose is $5 \mathrm{mg}$ at morning, but sometimes lower 2.5 or higher $7.5 \mathrm{mg}$ is given if needed.

Patients with secondary adrenal insufficiency should receive evaluation and adequate replacement for other pituitary hormone deficiencies. Replacement of thyroid hormone without replacement of glucocorticoid can precipitate acute adrenal insufficiency specially in hypopituitarism.

It is always advisable to use short acting steroid and lowest therapeutic dose to avoid steroid induced complications. Because, pharmacological dose is not always the therapeutic dose due to variable biological half-life of steroid. ${ }^{15}$ Sometime lower dose if advised to prevent iatrogenic Cushing's syndrome.

Patients should be well compliant and counseled properly. They should not miss dose rather increase at minor illness. Older patients are much vulnerable for adrenal crisis. Steroid is usually taken for whole life. May be withdrawn the drug in secondary adrenal insufficiency due to iatrogic adrenal insufficiency (steroid abuse) by close supervision under expert endocrinologist.

S. cortisol measures is not essential or helpful to adjust dose rather is shows always low level. ACTH is rarely done except in some cases like children with primary adrenal insufficiency or CAH.
Minerelocorticoid (fludrocortisones) is given usually $0.05-0.10 \mathrm{mg}$ daily if necessary. The adequacy is assessed by blood pressure, S. electrolytes and rarely plasma renin measurement. It may be required only with primary adrenal insufficiency. Care should be taken for overdose like hypernatremia, hypokalemia, uncontrolled hypertension etc.

Androgen replacement with DHEA usually $50 \mathrm{mg} /$ day in women occasionally who complained of decreased libido and fatigue and taking adequate dose of steroid. Care should be taken for side effects like acne, hirsutism.

Adrenal insufficiency, steroid and diabetes mellitus Prevalence of adrenal insufficiency seems to be higher in diabetic subjects. Though exact data is not available particularly in type 2 diabetes, many patients are being treated with steroid for adrenal disorder or for other diseases like Br. asthma, connective tissue disease, thyroiditis etc. But patients sometimes suffer from hypo or hyperglycemia. Frequent monitoring and frequent adjustment of dose preferably with basal bolus insulin regime is preferred. Metformin has some advantage to add on. Blood glucose is found usually higher at afternoon to evening in patients on maintenance dose of steroid.

\section{Steroid card}

Patients with glucocorticoid replacement more than 3 weeks should be counseled properly and provided with steroid card. The card includes instruction on emergency as well as minor illnesses like fever, vomiting etc. Patient should carry this all time which also includes, particulars of patient, phone number, diagnosis, type of steroid, dose and physician or hospital name. Sometime bracelet and even emergency pack containing disposable syringe, hydrocortisone injection $100 \mathrm{mg}$ specially for holidays/trip abroad. In minor illness, double the oral dose for few days only.

\section{Glucocorticoid withdrawal}

Usually withdrawal protocol is followed for steroid abusers suffering for chronic secondary adrenal insufficiency. But sometimes specially in ICU, patients may suffer from transient adrenal insufficiency and may be possible to withdraw steroid gradually. Patient on glucocorticoid of any dose more than 3 weeks should be withdrawn gradually by tapering the dose. ${ }^{13,20}$ All patients after withdrawal should be followed up by short 
Synacthen test to confirm the adequacy of adrenal function specially at stress or infection. In other cases (primary adrenal insufficiency and secondary adrenal insufficiency, other than drug induced) steroid should be continued lifelong under guidance of a experienced physician.

\section{Conclusion}

High degree of clinical suspicion is essential for diagnosis. Presentation may be endocrine, neurologic, cardiologic, dermatologic, gastroenterological, medical or electrolytes disturbances. Proper diagnosis and search for etiology is essential. Treatment should not be delayed in case of emergency while trying to confirm diagnosis and searching for etiology may be fatal. For ICU patients judging on the presently available clinical data, glucocorticoid cannot be randomly recommended as a routine adjuvant therapy, neither even in acute respiratory distress nor in all cases of septic shock. Glucocorticoid have, however, earned their spot among other rescue strategies in subgroups of ICU patients with the highest mortality risk. ${ }^{20}$ Considering clinical features and after proper evaluation of HPA axis steroid should be used. Steroid handling in an art, properly can be done by an experienced physician to reduce morbidity and mortality. Otherwise the steroid can itself cause more harm than benefit.

\section{References:}

1. Oelkers W. Adrenal insufficiency. N Engl J Med 1996; 335:1206.

2. Stuart PM, Krone NP. The Adrenal Cortex. In: Melmed S, Polonsky KS, Lersen PR, Kronenberg HM eds. Williums Text book of Endocrinology. 12 ${ }^{\text {th }}$ Edition. Philadelphia, Saunders 2011.pp479-544.

3. Addison T. On the constitutional and local effects of disease of the Supra-Renal capsules. London' Highley,1955.

4. Kong MF, Jeffcoate W. Eighty-six cases of Addison's disease. Clin Endocrinol (Oxf) 1994; 41:757-61.

5. Barnett AH, Espiner EA, Donald RA. Patients presenting with Addison's disease need not be pigmented. Postgrad Med J 1982; 58:690.

6. UpToDate. Topic 154 Version 16.0. 2014.(cited Nov 01, 2014)

7. Galbois A, Rudler M, Massard J et al. Assessment of adrenal function in cirrhotic patients: salivary cortisol should be preferred. J Hepatol 2010; 52:839.
8. Klose M, Lange M, Rasmussen AK et al. Factors influencing the adrenocorticotropin test: role of contemporary cortisol assays, body composition, and oral contraceptive agents. J Clin Endocrinol Metab 2007; 92:1326.

9. Florkowski CM, Holmes SJ, Elliot JR et al. Bone mineral density is reduced in female but not male subjects with Addison's disease. N Z Med J 1994; 107:52.

10. Valero MA, Leon M, Ruiz Valdepeñas MP et al. Bone density and turnover in Addison's disease: effect of glucocorticoid treatment. Bone Miner 1994; 26:9.

11. Løvås $\mathrm{K}, \mathrm{Gjesdal} \mathrm{CG}, \mathrm{Christensen} \mathrm{M}$ et al. Glucocorticoid replacement therapy and pharmacogenetics in Addison's disease: effects on bone. Eur J Endocrinol 2009; 160:993.

12. Suliman AM, Freaney R, Smith TP et al. The impact of different glucocorticoid replacement schedules on bone turnover and insulin sensitivity in patients with adrenal insufficiency. Clin Endocrinol (Oxf) 2003; 59:380.

13. Streeten DH, Anderson GH Jr, Bonaventura MM. The potential for serious consequences from misinterpreting normal responses to the rapid adrenocorticotropin test. J Clin Endocrinol Metab 1996; 81:285.

14. Hamrahian AH, Oseni TS, Arafah BM. Measurements of serum free cortisol in critically ill patients. N Engl J Med 2004; 350:1629-38.

15. Dickstein G, Shechner C, NicholsonWE, Rosner I, ShenOrr Z, Adawi $\mathrm{F}$ et al. Adrenocorticotropin stimulation test: effect of basal cortisol level, time of day, and suggested new sensitive low dose test. J Clin Endocrinol Metab 1991;72:773-78.

16. Cooper MS, Stewart PM. Adrenal insufficiency in critical illness. J Intensive Care Med 2007;22: 348-62.

17. Rothwell PM, Lawler PG. Prediction of outcome in intensive care patients using endocrineparameters. Crit Care Med 1995;23: 78-83.

18. Annane D, Sebille V, Troche G, Raphael JC, Gajdos P, Bellisant E. A 3-level prognostic classification in septic shock based on cortisol level and cortisol response to corticotropin. JAMA 2000; 283:1038-45.

19. Michels AW, Eisenbarth GS. Autoimmune polyendocrine syndrome type 1 (APS-1) as a model for understanding autoimmune polyendocrine syndrome type 2 (APS-2). $J$. Intern. Med 2009; 265, 530-40.

20. Vassiliadi DA, Dimopoulou I, Tzanela M, Douka E, Livaditi $\mathrm{O}$, Orfanos SE et al. Longitudinal assessment of adrenal function in the early and prolonged phases of critical illness in septic patients: relations to cytokine levels and outcome. J Clin Endocrinol Metab 2014 Aug. 22:jc20142619. (cited Nov 1, 2014). 